\title{
Abnormal cortical excitability in sporadic but not homozygous D90A SOD1 ALS
}

\author{
M R Turner, A D Osei-Lah, A Hammers, A Al-Chalabi, C E Shaw, P M Andersen, D J Brooks, \\ P N Leigh, K R Mills
}

J Neurol Neurosurg Psychiatry 2005;76:1279-1285. doi: 10.1136/jnnp.2004.054429

See end of article for authors' affiliations

\section{Correspondence to:}

Correspondence to:
Dr M R Turner, PO Box 41

(ANC), Institute of

Psychiatry, De Crespigny

Park, London SE5 8AF,

UK; m.turner@iop.kcl.

ac.uk

Received

19 September 2004

In revised form

14 November 2004

Accepted

20 December 2004

Background: Excitotoxicity is one pathogenic mechanism proposed in amyotrophic lateral sclerosis (ALS), and loss of cortical inhibitory influence may be contributory. Patients with ALS who are homozygous for the D90A superoxide dismutase-1 (SOD1) gene mutation (homD90A) have a unique phenotype, associated with prolonged survival compared with patients with sporadic ALS (sALS). In this study, transcranial magnetic stimulation (TMS) was used to explore cortical excitation and inhibition. Flumazenil binds to the benzodiazepine subunit of the $\mathrm{GABA}_{\mathrm{A}}$ receptor, and ${ }^{11} \mathrm{C}$-flumazenil positron emission tomography (PET) was used as a marker of cortical neuronal loss and/or dysfunction, which might in turn reflect changes in cortical inhibitory GABAergic mechanisms.

Methods: Cortical responses to single and paired stimulus TMS were compared in 28 patients with sALS and 11 homD90A patients versus 24 controls. TMS measures included resting motor threshold, central motor conduction time, silent period, intracortical inhibition (ICI), and facilitation. ${ }^{11} \mathrm{C}$-flumazenil PET of the brain was performed on 20 patients with sALS and nine with homD90A. Statistical parametric mapping was used to directly compare PET images from the two patient groups to identify those areas of relatively reduced cortical ${ }^{11} \mathrm{C}$-flumazenil binding that might explain differences in cortical excitability seen using TMS.

Results: Increased cortical excitability, demonstrated by reduction in $\mathrm{ICl}$, was seen in the patients with sALS but not the homD90A patients. A relative reduction in cortical ${ }^{11} \mathrm{C}$-flumazenil binding was found in the motor and motor association regions of the superior parietal cortices of the patients with sALS.

Conclusions: A cortical inhibitory deficit in sALS was not demonstrable in a homogeneous genetic ALS population of similar disability, suggesting a distinct cortical vulnerability. ${ }^{11}$ C-flumazenil PET demonstrated that neuronal loss/dysfunction in motor and motor association areas may underlie this difference. The corollary, that there may be relative preservation of neuronal function in these areas in the homD90A group, has implications for understanding the slower progression of disease in these patients.

A myotrophic lateral sclerosis (ALS) is characterised by degeneration of corticospinal, brain stem, and spinal cord motor neurones ${ }^{1}$ The cause of ALS remains uncertain. It is established that the cortical lesion is widespread, and is not confined to motor regions..$^{2-4}$

Transcranial magnetic stimulation (TMS) allows cortical and corticospinal pathway function and integrity to be probed non-invasively, ${ }^{5}$ and when used with the triple stimulation technique, ${ }^{6}$ has proved useful in characterising the degree of upper motor neurone (UMN) involvement in ALS, which is variable. Hyperexcitability of the motor cortex as revealed by TMS could reflect raised intrinsic excitability of cortical cells, or a diminished inhibition of these cells. Paired TMS, using a subthreshold conditioning stimulus followed 1-5 milliseconds later by a suprathreshold test stimulus, provides unique insight into the function of intracortical inhibitory circuits, ${ }^{7}$ mediated through $\gamma$-amino butyric acid (GABA)ergic transmission. At longer (7-50 ms) interstimulus intervals (ISIs), the test response is facilitated. Such studies in ALS have provided evidence of increased cortical excitability, ${ }^{8-10}$ and support a role for excitotoxic pathogenic mechanisms. ${ }^{11}$ Increased cortical excitability may be caused either by increased excitability, or decreased inhibition, of neuronal pathways.

Flumazenil binds to the benzodiazepine subunit of the $\mathrm{GABA}_{\mathrm{A}}$ receptor present on neurones throughout the cerebral cortex. ${ }^{11} \mathrm{C}$-flumazenil positron emission tomography (PET) therefore provides a marker for cortical neuronal loss and/or dysfunction that might also reflect altered GABA-ergic inhibitory function. A pilot study with this PET radioligand confirmed significant cortical binding decreases in patients with ALS compared with controls, and not confined to motor regions. ${ }^{12}$

About $5-10 \%$ of ALS cases are associated with mutations of the superoxide dismutase-l (SODI) gene on chromosome $21 .^{13-15}$ Most of the 109 known SOD1 gene mutations are associated with variable phenotypes, a survival period typically in the order of 1-4 years, and are inherited as a dominant trait. ${ }^{14}$ The exception is the most prevalent SODI mutation worldwide, an aspartate $\rightarrow$ alanine substitution at codon 90 (D90A). The D90A SODI gene mutation is usually inherited as a recessive trait. There is an invariable phenotype (beginning with lower limb spasticity and weakness, prior to upper limb and bulbar involvement), and prolonged survival (mean 14 years). ${ }^{16}$ Worldwide, patients with D90A have been shown to share a common founder some 18000 years ago.

Abbreviations: ALS, amyotrophic lateral sclerosis; ALSFRS-R, ALS functional rating scale; CMAP, compound muscle action potential; $\mathrm{CMCT}$, central motor conduction time; FDI, first dorsal interosseous; GABA, gamma aminobutyric acid; ICF, intracortical facilitation; ICI, intracortical inhibition; ISI, interstimulus intervals; MEP, motor evoked potentials; PET, positron emission tomography; PSTH, peristimulus time histogram; RMT, resting motor threshold; sALS, sporadic amyotrophic lateral sclerosis; SOD1, superoxide dismutase-1; SP, silent period; S-R, stimulus response; TMS, transcranial magnetic stimulation; UMN, upper motor neurone; VD, volume of distribution 
To explain this paradox, the existence of a linked protective factor has been postulated, but the neuroprotective mechanism of this factor is not understood. ${ }^{16-18}$ A TMS study in patients with ALS homozygous for D90A, also aimed at exploring inhibitory mechanisms, employed peristimulus time histograms (PSTHs) rather than paired pulse stimuli. The authors were able to demonstrate delayed peak responses and a "suppression phase" in the patients with D90A compared with those with sporadic ALS, which they concluded was evidence of preserved cortical inhibitory influence. ${ }^{19}$

Using TMS, the resting motor threshold (RMT), central motor conduction time (CMCT), stimulus response (S-R) curves, silent period (SP) duration, and intracortical inhibition (ICI) and facilitation (ICF) were evaluated in a group of patients with sporadic ALS (sALS), and a group of affected patients homozygous for D90A (homD90A). The results were compared with findings in a group of healthy controls. In addition, ${ }^{11} \mathrm{C}$-flumazenil PET was performed on subjects from both patient groups, comparing them with each other to look for relative regional reductions in cortical ${ }^{11} \mathrm{C}$-flumazenil binding that might reflect reduced $\mathrm{GABA}_{\mathrm{A}}$ receptor function.

\section{METHODS}

\section{Participants}

All patients were diagnosed with ALS according to revised El Escorial criteria. ${ }^{20}$ Affected patients were rated functionally using the revised ALS functional rating scale (ALSFRS-R; $0-48$, lower scores correlate with increasing disability). ${ }^{21}$ Patients were also graded in terms of UMN "burden", by totaling the number of UMN signs on examination. These were taken as pathologically brisk biceps, supinator, triceps, finger, knee, and ankle reflexes, and extensor plantar responses assessed bilaterally, and brisk facial and jaw jerks (maximum possible score 16) (see also Turner $e t a l^{4}$ ). Disease duration at the time of investigation was calculated in months from date of first symptom onset to date of investigation.

All subjects were asked to omit all medication for at least 48 hours prior to testing. None was taking any regular medication known to interact with the benzodiazepine receptor. All patients gave informed written consent, and the study was approved by the ethics committees of the Institute of Psychiatry, Hammersmith Hospitals NHS Trust, Umeå University, and King's College Hospitals.

\section{TMS studies}

Surface electromyographic activity was recorded from the right first dorsal interosseous (FDI) muscle. The compound muscle action potential (CMAP) was determined by supramaximal electrical stimulation of the ulnar nerve at the wrist. Magnetic cortical stimulation was delivered from a Magstim 200 device (Magstim Co. Ltd, Dyfed, UK) driving a circular coil centred at the vertex and held tangentially to excite preferentially the left hemisphere. F wave minimal latency was determined from 10 consecutive supramaximal stimuli of the ulnar nerve at the right wrist at $1 \mathrm{~Hz}$.

The mean RMT was determined using previously described methods. ${ }^{22}$ The S-R curves were evaluated by stimulating the motor cortex at 0.9, 1.0, 1.1, 1.2, and 1.3 times threshold, initially with the muscle at rest (auditory feedback was provided) and then with a modest isometric contraction of the FDI muscle.

ICI and ICF were evaluated using paired pulse stimulation. The intensity of the conditioning stimulus was set at 0.9 times threshold and the test stimulus set at 1.3 times threshold. Paired pulse stimulation was then performed, in the resting state, using a BiStim device (Magstim Co.) capable of delivering the two stimuli at varying ISIs $(1,2,3$, $4,5,7,10,20$, and $50 \mathrm{~ms}$ ).

Ten trials were collected for each paradigm, whose order was also randomised. The area of the averaged rectified motor evoked potentials (MEPs) obtained under each experimental condition was the measure of interest. For analysis of the S-R curves, the area of the averaged MEP obtained at each stimulus intensity was expressed as a percentage of the area of the peripherally evoked CMAP.

The SP duration was measured in each trial from the stimulus artefact to the onset of the return of sustained voluntary EMG activity, by visual inspection, using a gain of $100 \mu \mathrm{V} /$ division. The average of 10 trials was calculated for each stimulus intensity.

To analyse ICI and ICF, the mean area of the conditioned MEP obtained at each ISI was expressed as a percentage of the mean area of 30 unconditioned (test) MEPs. (inhibition $<100$, facilitation $>100)$. Central motor conduction time (CMCT) was determined using the F wave method. ${ }^{23}$

\section{Statistical analysis}

All statistical analyses were performed using SPSS software (version 11.0; SPSS Inc., Chicago, IL, USA). One way analysis of variance was performed for all TMS variables with post hoc analysis comparing patient groups with controls (two sided Dunnett test to correct for multiple comparisons). Results with $\mathrm{p}<0.05$ were considered significant.

In order to explore various combinations of three or more consecutive ISIs from the paired data (for example, 1-3, 1-4, 5-10 ms), a repeated measures analysis of variance was used, with identical post hoc group analysis, comparing affected patient groups with controls (and with adjustment for multiple comparisons), with significance again set at $\mathrm{p}<0.05$.

Two tailed bivariate correlations, with a Pearson coefficient, were examined between clinical variables (ALSFRS-R, duration of disease, and UMN score) and TMS measures for both affected patient groups separately. One way analysis of variance was performed for both patient groups using sex and site of onset (the latter only used for the sALS group, as the homD90A group were all lower limb onset), against all TMS measures. For the control group, similar analyses were carried out for age and sx against all TMS measures.

To reduce the potential for a type I error, only those correlations with a very high correlation coefficient (greater than $+/-0.7)$ and $\mathrm{p}<0.05$ were considered potentially relevant. However any firm conclusions drawn from these correlational results must still remain cautious, as they were not subject to any correction for potential error due to multiple comparisons.

\section{PET studies}

${ }^{11} \mathrm{C}$-flumazenil PET images were acquired in three dimensional mode using an ECAT 953B PET scanner (CTI/Siemens, Knoxville, TN, USA), in 20 frames over 90 minutes. Scanning started 30 seconds before a bolus injection of approximately $10 \mathrm{mCi}(370 \mathrm{MBq}){ }^{11} \mathrm{C}$-flumazenil intravenously over $30 \mathrm{sec}-$ onds into an antecubital fossa vein. A metabolite corrected arterial plasma input function and spectral analysis ${ }^{24}$ were used to derive parametric images of volume of distribution (VD) for the ligand.

Statistical parametric mapping (SPM'99; Wellcome Department of Cognitive Neurology) was used to make group comparisons of the normalised and smoothed VD images. ${ }^{25}$ Individual VD images were normalised into standard Montreal Neurological Institute space using a normal ${ }^{11} \mathrm{C}$ flumazenil template created in house and a $t$ test applied at voxel level to mean VD images from controls and from patients with ALS. A proportional voxel threshold of 0.8 (default setting) was used. Proportional scaling of the global 
VD was used (calculated using the default setting). An overall image threshold of $p=0.05$ was applied to the SPMs, and only those clusters identified with a corrected $\mathrm{p}<0.05$ were displayed.

\section{RESULTS}

\section{Missing data}

Clinical variables were incomplete in two of the patients with sALS. Four of the sALS and two of the homD90A patients had thresholds greater than the maximum output of the stimulator. There was no obvious phenotypic difference between these and other patients in the respective groups, and their threshold was designated $100 \%$ for the subsequent analyses. The CMCT result was missing from a total of five subjects with sALS and two homD90A subjects, owing to failure to complete the protocol or recording artefacts preventing reliable measure.

\section{Participants}

The group characteristics in the TMS studies are summarised in table $1 .{ }^{11} \mathrm{C}$-flumazenil PET was performed on 20 of the 28 patients with sALS (mean age 57 years, ALSFRS-R 38, UMN score 8 ) and nine of the 11 homD90A patients (mean age 53 years, ALSFRS-R 33, UMN score 8).

TMS studies, RMT and CMCT, S-R curves, and SP The mean and SD for each measure in the TMS studies are shown in table 2.

RMT and CMCT were both significantly higher than controls in both the sALS and homD90A groups $(p<0.05)$ (fig l).

There were no significant group differences in the S-R curves, obtained with the target muscle relaxed. When the target muscle was contracted, the homD90A patients showed significantly reduced MEP size when the stimulus intensity was 1.3 times threshold, compared with controls $(p<0.05)$ (fig 2).

There was no significant difference in the duration of the SP for any group (fig 3).

\section{Inhibitory measures}

In the paired stimulus paradigm, the sALS patient group showed significantly reduced inhibition at ISI 1 and $2 \mathrm{~ms}$ and reduced facilitation at 10 and $50 \mathrm{~ms}(\mathrm{p}<0.05)$. The mean inhibition ratio across the range of ISI $1-3 \mathrm{~ms}$ was also significantly reduced in the sALS group compared with controls $(p<0.05)$, but not using any other combination of multiple ISIs. In contrast, the pattern of inhibitory and facilitatory responses across the full range of inidividual ISIs in the homD90A group was not significantly different from controls (fig 4).

\begin{tabular}{|c|c|c|c|}
\hline Variable & sALS & homD90A & Control \\
\hline $\mathrm{n}$ & 28 & 11 & 24 \\
\hline $\operatorname{Sex}(M: F)$ & $21: 7$ & $3: 8$ & $13: 11$ \\
\hline Age & $\begin{array}{c}58(9) \\
9\end{array}$ & $\begin{array}{l}52(12) \\
12\end{array}$ & $\begin{array}{l}41(13) \\
13\end{array}$ \\
\hline ALSFRS-R & $37(5)$ & 34 (8) & - \\
\hline & & 8 & - \\
\hline UMN score & $8(6)$ & $9(3)$ & NA \\
\hline Duration (months) & $\begin{array}{l}6 \\
29(18) \\
18\end{array}$ & $\begin{array}{l}3 \\
65(57) \\
57\end{array}$ & NA \\
\hline \multicolumn{4}{|c|}{$\begin{array}{l}\text { ALSFRS-R, revised amyotrophic lateral sclerosis functional } \\
\text { rating scale; homD } 90 A \text {, homozygous D90A SODI ALS; } \\
\text { SALS, sporadic amyotrophic lateral sclerosis; UMN, upper } \\
\text { motor neurone. Values are mean (SD where applicable). }\end{array}$} \\
\hline
\end{tabular}
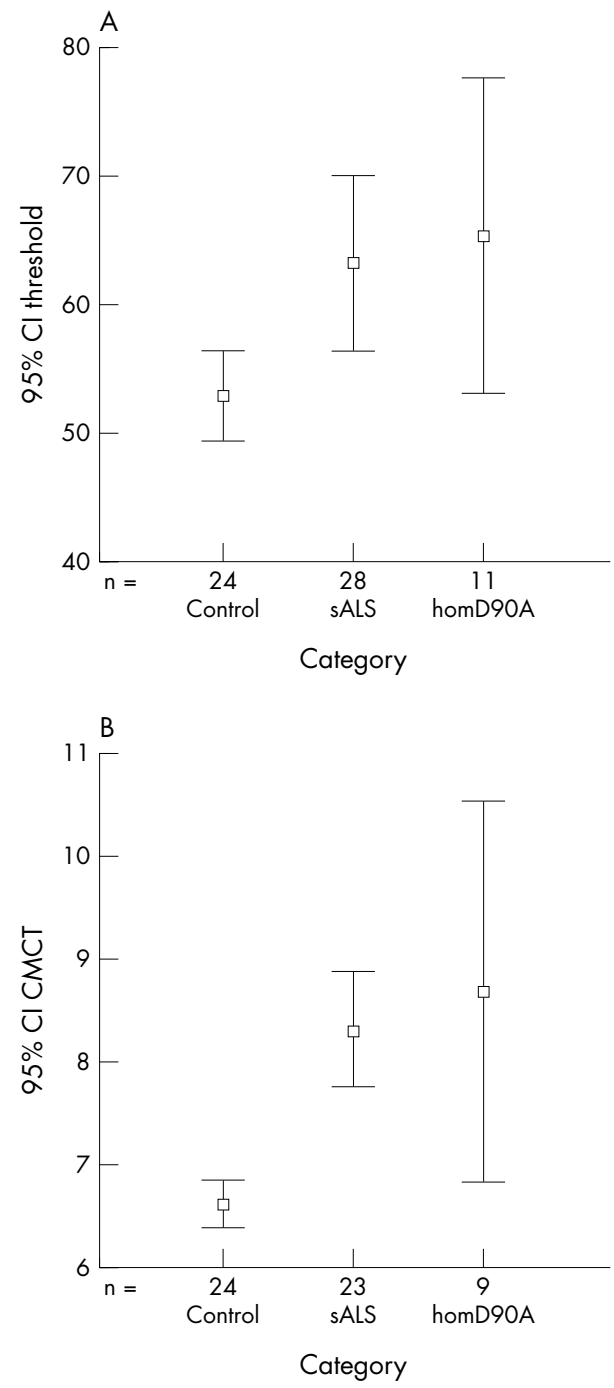

Figure 1 Error bar plots with 95\% confidence intervals (Cls) of RMT (A) and CMCT (B) for each subject group. Both measures are significantly higher in the patient groups compared with controls $(p<0.05)$. RMT, resting motor threshold; CMCT, central motor conduction time; sALS, sporadic ALS; homD90A, homozygous D90A SODI ALS.

\section{Intragroup correlations}

In the control population there was no significant correlation between age at investigation and any TMS measure, and no significant effect of sex.

Within the sALS group paired study at ISI $1 \mathrm{~ms}$, men showed significantly greater inhibition than women (37\% $v$ $84 \%$ of unconditioned response, $\mathrm{p}<0.02)$. There was no significant effect of sex, site of onset, or clinical variables such as UMN score, for any other TMS measures.

Within the homD90A group, duration of disease correlated positively with motor stimulation threshold $(\mathrm{r}=0.8$, $\mathrm{p}<0.004)$ and size of the conditioned response at ISI $3 \mathrm{~ms}$ $(\mathrm{r}=1, \mathrm{p}<0.001)$, and negatively with duration of the silent period when the stimulus intensity was threshold and 1.1 and 1.2 times threshold $(\mathrm{r}>-0.7, \mathrm{p}<0.02)$. The ALSFRS-R score correlated negatively with the size of the inhibitory response at ISI $1 \mathrm{~ms} \quad(\mathrm{r}=-0.9, \mathrm{p}=0.003)$ and $3 \mathrm{~ms}$ $(\mathrm{r}=-0.8, \mathrm{p}<0.01)$. The UMN score correlated negatively with size of the MEP obtained when the stimulus intensity was 1.1 times threshold with the target muscle relaxed $(\mathrm{r}=-0.7, \mathrm{p}<0.03)$. There was no significant effect of sex. 

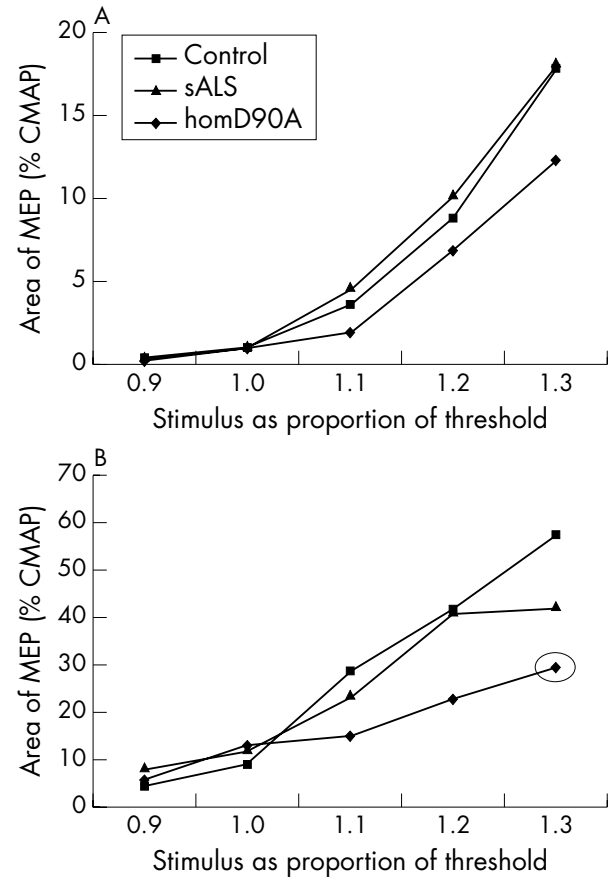

Figure 2 Responses to TMS across a range of stimulus strengths with the target muscle relaxed (A) and activated (B). The homD90A group showed significantly less response at maximum stimulation in the activated state compared with controls $(p<0.05)$ (result circled). sALS, sporadic ALS; homD90A, homozygous D90A SODI ALS; MEP, motor evoked potential; CMAP, compound motor action potential; $t$, threshold.

\section{PET studies}

Bilateral significant areas of relatively decreased cortical binding of ${ }^{11} \mathrm{C}$-flumazenil to $\mathrm{GABA}_{\mathrm{A}}$ receptors were seen in the sALS patients compared with the homD90A subjects $(p<0.05)$. These were concentrated over the motor cortex (precentral gyri) and posteriorly in the motor association areas, particularly over the superior parietal cortices (fig 5).

\section{DISCUSSION}

This TMS study has provided evidence of differential cortical excitability in patients with sALS and those with SLS and homD90A SOD1 with similar functional disability. A unique parallel visualisation of the $\mathrm{GABA}_{\mathrm{A}}$ receptor through ${ }^{11} \mathrm{C}$ flumazenil PET localised the relative deficit in the sALS patients to motor and motor association areas. Although we cannot be certain whether the reductions in binding of ${ }^{11} \mathrm{C}$ flumazenil are due to neuronal loss or purely functional GABA-ergic decreases, the two are not mutually exclusive.

Patients with sALS showed evidence of reduced intracortical inhibition in paired pulse studies at short ISIs, but also a significant lack of facilitation at higher ISIs, compared with controls. In contrast, paired pulse studies in patients with homD90A demonstrated preserved inhibition. The pattern of relatively reduced cortical ${ }^{11} \mathrm{C}$-flumazenil binding in patients with sALS compared with the homD90A patients supports the concept of reduced inhibitory influence, via $\mathrm{GABA}_{\mathrm{A}}$ receptor transmission, in sALS. The relative preservation of such circuitry in homD90A patients with ALS may be one mechanism accounting for prolonged survival in this group.

\section{Cortical excitability in ALS and previous TMS studies} The concept of excitotoxic neuronal damage in ALS (and other neurodegenerative disorders) is established. ${ }^{1126} \mathrm{~A}$ contributory mechanism may be loss of inhibitory GABAergic function, and pathological studies support loss
Table 2 TMS measures in controls and patient groups

\begin{tabular}{|c|c|c|c|}
\hline TMS variable & Control & sALS & homD90A \\
\hline Threshold & $53(8)$ & $63(17)^{*}$ & $65(18)^{*}$ \\
\hline CMCT & 7 (1) & $8(1)^{*}$ & $9(2)^{*}$ \\
\hline Area R 0.9 † & $0(1)$ & $0(1)$ & $0(0)$ \\
\hline Area R 1.0 t & $1(2)$ & $1(1)$ & $1(1)$ \\
\hline Area R $1.1 \dagger$ & $4(4)$ & $5(6)$ & $2(2)$ \\
\hline Area R $1.2 \mathrm{t}$ & $9(10)$ & 10 (10) & $7(8)$ \\
\hline Area $\mathrm{R} 1.3 \dagger$ & $18(16)$ & 18 (19) & $12(14)$ \\
\hline Area A $0.9+$ & $4(5)$ & $8(10)$ & $6(11)$ \\
\hline Area A $1.0 \mathrm{t}$ & $9(10)$ & $12(15)$ & $13(12)$ \\
\hline Area A $1.1 \dagger$ & $29(25)$ & $24(22)$ & $15(12)$ \\
\hline Area A $1.2 \mathrm{t}$ & $42(22)$ & $41(36)$ & 23 (16) \\
\hline Area A $1.3 \mathrm{t}$ & $57(24)$ & 42 (39) & $30(19)^{*}$ \\
\hline SP duration $0.9 \mathrm{t}$ & $72(24)$ & $94(45)$ & $86(39)$ \\
\hline SP duration $1.0 \mathrm{t}$ & $99(27)$ & $113(44)$ & $120(50)$ \\
\hline SP duration $1.1 \mathrm{t}$ & $136(33)$ & $140(48)$ & $131(56)$ \\
\hline $\mathrm{SP}$ duration $1.2 \mathrm{t}$ & $156(27)$ & $160(53)$ & 151 (67) \\
\hline SP duration $1.3 \mathrm{t}$ & 169 (32) & $177(57)$ & $155(67)$ \\
\hline Area ISI $1 \mathrm{~ms}$ & $22(21)$ & $49(44)^{*}$ & 31 (33) \\
\hline Area ISI $2 \mathrm{~ms}$ & $23(26)$ & $51(58)^{*}$ & $15(16)$ \\
\hline Area ISI $3 \mathrm{~ms}$ & $39(56)$ & 71 (104) & 31 (29) \\
\hline Area ISI $4 \mathrm{~ms}$ & $48(45)$ & 105 (238) & $18(18)$ \\
\hline Area ISI $5 \mathrm{~ms}$ & $84(65)$ & 92 (131) & 38 (39) \\
\hline Area ISI $7 \mathrm{~ms}$ & $155(130)$ & $123(90)$ & 97 (45) \\
\hline Area ISI $10 \mathrm{~ms}$ & $239(206)$ & $136(80)^{*}$ & 144 (123) \\
\hline Area ISI $20 \mathrm{~ms}$ & 161 (202) & $126(83)$ & 171 (184) \\
\hline Area ISI $50 \mathrm{~ms}$ & $141(94)$ & $66(66)^{*}$ & $116(63)$ \\
\hline
\end{tabular}

Values are mean (SD). Areas R and A are \% CMAP; area ISI values are \% unconditioned. *Significant group differences from controls $(p<0.05)$. A, muscle in activated state; CMAP, compound motor action potential; $\mathrm{CMCT}$, central motor conduction time; homD90A, homozygous D90A SOD1 ALS; ISI, interstimulus interval; $R$, muscle in relaxed state; sALS, sporadic amyotrophic lateral sclerosis; SP, silent period; $t$, threshold; TMS, transcranial magnetic stimulation.

of cerebral inhibitory interneuronal cells in $\mathrm{ALS}^{3}$ and an alteration in the expression of $\mathrm{GABA}_{\mathrm{A}}$ receptor unit subtypes in the motor cortex..$^{27} \mathrm{~A}$ previous ${ }^{11} \mathrm{C}$-flumazenil PET study supported loss of cortical GABAergic influence in ALS, possibly through loss of neurones bearing the receptor, and this was not confined to motor regions. ${ }^{12}$ PET activation studies support the concept of increased cortical excitability through a "boundary shift" effect during motor activity. ${ }^{28}$ In keeping with our findings, several other studies in patients with sporadic ALS have demonstrated a deficit of intracortical inhibition at short ISIs, suggestive of increased cortical excitability. ${ }^{8-10}$

In contrast to a previous study, ${ }^{9}$ we demonstrated significantly reduced facilitation at 10 and $50 \mathrm{~ms}$ ISIs in the patients with sALS compared with controls. Although

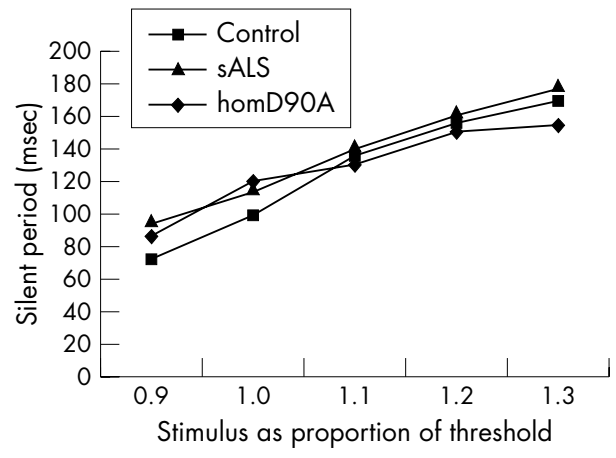

Figure 3 Silent period measured during stimulation with the target muscle in the activated state. There was a lengthening of SP with increasing stimulus strength, but no significant difference between patient groups and controls. sALS, sporadic ALS; homD90A, homozygous D90A SOD1 ALS; ms, milliseconds; t, threshold. 


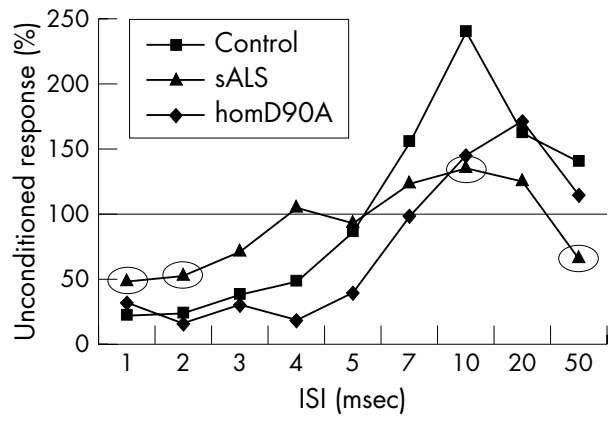

Figure 4 Inhibition of response to paired TMS stimuli over a range of interstimulus intervals, expressed as a percentage of the unconditioned response (lower percentage $=$ more inhibition/less facilitation; $100 \%$ line drawn horizontally). The sALS group shows significantly reduced inhibition compared with controls at 1 and $3 \mathrm{~ms}$, and significantly reduced facilitation compared with controls at 10 and $50 \mathrm{~ms}(p<0.05)$ (results circled). The homD90A group responses were not significantly different to those of the controls however, suggesting relative preservation of inhibitory cortical mechanisms. ICl, intracortical inhibition. ICF, intracortical facilitation; ISI, interstimulus interval; sALS, sporadic ALS; homD90A, homozygous D90A SODI ALS; ms, milliseconds.

facilitation was not statistically significantly reduced in the homD90A patients, this may simply reflect the greater variance in the data for this group. Riluzole has been reported to alter TMS measures both in patients with ALS and in controls. It does not appear to alter motor threshold, ${ }^{29}$ but may increase the duration of the $\mathrm{SP}^{30}$ and enhance ICI in patients. $^{3031}$ Although it was found to suppress ICF in controls, ${ }^{32}{ }^{33}$ this was not demonstrated in patients. ${ }^{31}$ It was not clearly stated whether riluzole was being taken by the patients tested in previous paired stimulus TMS studies in $\mathrm{ALS}^{8-10}$; however, it is unlikely, as the licensing of this drug occurred near to the time that these studies were performed. Although in the present study patients did not take riluzole for at least 48 hours prior to study (at least four drug half lives $^{34}$ ), it is possible that some effect of this drug persisted, and theoretically this could account for the relatively reduced facilitatory responses in our results. However, another preriluzole study also noted reduced ICF in some ALS patients. ${ }^{35}$ We are confident that the differences in ICI are not merely a drug effect; indeed, given that riluzole might be expected to have enhanced ICI, the size of the differences in this study would then be an underestimate. In a study of the effects of carbemazepine on TMS measures, no effect on ICI or ICF was found. ${ }^{36}$

The most striking finding is the lack of significant difference in response to paired stimulation in the homD90A patients with ALS compared with controls. This strongly suggests that inhibitory pathways responsible for the effect at short ISIs are intact in this patient group, in contrast to the patients with sALS. It is noteworthy that this was in spite of the fact that the homD90A patients were functionally slightly more disabled than the patients with sALS (mean ALSFRS-R score $34 v 37$ ).

Such inhibitory pathways may conceivably also have a role in the significantly reduced response to high intensity stimulation in the activated paradigm that we noted in the homD90A group compared with controls, but this remains speculative. We noted a correlation at short ISIs between reduced inhibitory responses and both increased disease duration and decreased ALSFRS-R score, suggesting that the relative preservation of cortical inhibitory function in the homD90A patients may wane over the course of the disease in this group.

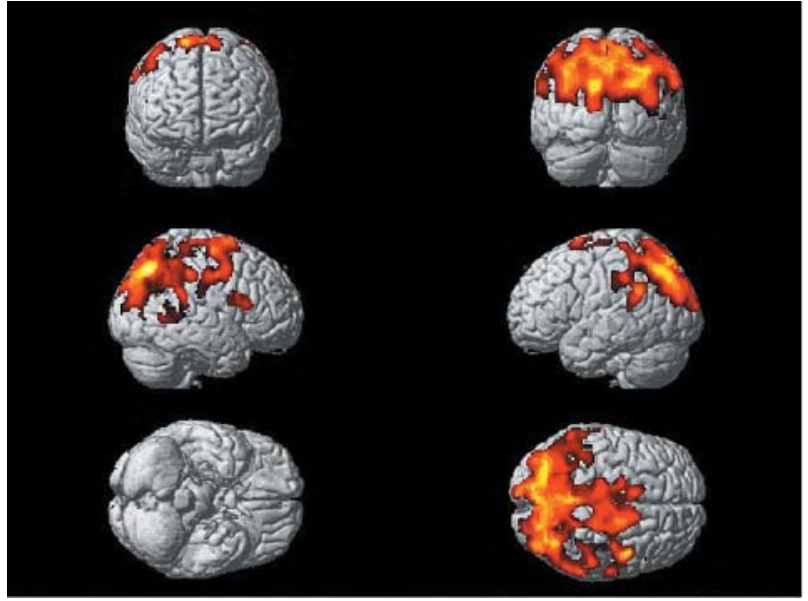

Figure 5 SPM rendered to generic 3D brain (shown from six angles) demonstrating areas of relative decrease of ${ }^{11} \mathrm{C}$-flumazenil binding in patients with sALS versus those with homD90A (cluster corrected $p<0.05$ ). The areas of relatively decreased binding in the sALS patients were concentrated in the motor (precentral gyri) and motor association areas, particularly those of the superior parietal lobes bilaterally. This relative reduction may reflect the increased cortical excitability seen during TMS, in contrast to the relatively preserved inhibition in the homD90A patients. sALS, sporadic ALS; homD90A, homozygous D90A SODI ALS

The striking relative reduction in sALS compared with the homD90A group for the binding of ${ }^{11} \mathrm{C}$-flumazenil to $\mathrm{GABA}_{\mathrm{A}}$ receptors in motor and motor association areas is compatible with reduced GABAergic influence, either through loss of neurones bearing the receptor or functional receptor changes, or indeed both. It is established that patients with sporadic ALS have significant cortical reduction in the binding of ${ }^{11} \mathrm{C}$-flumazenil compared with healthy controls. ${ }^{12}$ The involvement of parietal regions might seem surprising, but neuronal changes in this area have been previously noted in neuropathological ${ }^{237}$ and neuroimaging ${ }^{38}$ studies in ALS. The relative preservation of inhibitory influences in such areas in the homD90A patients may be linked to the preserved inhibition seen neurophysiologically, ${ }^{19}$ and suggests an altered cortical vulnerability in this group.

\section{Central motor conduction time and RMT}

Our study demonstrated significantly prolonged CMCT and RMT in both the sALS and homD90A patient groups compared with controls. Prolongation of the CMCT in sALS has been previously reported to occur in only about $20 \%$ of sALS patients, ${ }^{39-42}$ but was more consistently seen in a study of homD90A patients. ${ }^{16}$ In this latter group, however, it has been shown that cortex to muscle latency may only be prolonged when the muscle is activated. ${ }^{43}$ CMCT is not thought to change with disease progression, ${ }^{39}$ and there was no correlation between this measure and disease duration in this study.

Some studies have noted a reduced corticomotor threshold in ALS, frequently soon after onset of symptoms. ${ }^{22}{ }^{44}$ We chose to include those patients with a threshold above maximum possible stimulation in the analysis (designated $100 \%)$, and although their exclusion rendered the differences in mean RMT non-significant, it was still not lower than controls in either group. It has been previously reported that there is no change in RMT with disease progression in SALS, ${ }^{39} 45$ although we noted a positive correlation in the homD90A group. 


\section{Silent period}

The SP is, in part, a measure of cortical inhibition, ${ }^{7}$ and has been reported to be shortened in patients with ALS, ${ }^{41}$ and to decrease with disease progression. ${ }^{46}{ }^{47}$ In a more detailed study it was suggested that any reduction is only seen shortly after clinical presentation, and is less obvious as the disease evolves. ${ }^{39}$ Although there was a steady increase in SP with increasing stimulus intensity in our study, this occurred in all groups. The disease duration in this study ranged from 9 to 80 months in the sALS and 9 to 162 months in the homD90A group, and so was probably beyond the "early stages" where changes in SP might have been seen. There were, however, negative correlations noted between disease duration and SP length in the homD90A group at two stimulus strengths, but not the patients with sALS.

\section{UMN involvement}

In order to qualify for one of the defined categories within the revised El Escorial criteria for ALS, ${ }^{20}$ it is necessary to have demonstrable UMN signs. This study incorporated a 16 point scale of pathological UMN signs, which lends itself easily to parametric analysis. In a study of microglial activation in vivo, the UMN score correlated closely with microglial activation in the motor cortex. ${ }^{4}$ Previous studies have noted higher motor stimulation thresholds in those with UMN signs in the hands, ${ }^{39} 48$ and lower than normal thresholds in those with no signs in the hands. ${ }^{22} 44$

The mean UMN score was similar for both patient groups in this study, and no meaningful correlations between UMN score and threshold, CMCT, or any other TMS measures were noted; neither was there a significant effect of site of onset within the sALS group. In contrast to imaging studies, where the UMN score has correlated with increased burden of cortical disease, ${ }^{49}$ overall the influence of UMN involvement on the cortical lesion as demonstrated by TMS remains unclear.

\section{Sex}

In keeping with another study,,$^{50}$ no clear effect of sex on TMS measures in controls was demonstrated.

\section{CONCLUSION}

This study supports the notion that cortical excitability is abnormally increased in sALS, and also demonstrates that patients with similar functional disability but homozygous for the D90A SODI gene mutation appear to have relatively preserved cortical inhibitory mechanisms. The relatively reduced binding of the PET $\mathrm{GABA}_{\mathrm{A}}$ receptor ligand ${ }^{11} \mathrm{C}$ flumazenil in motor and motor association cortical regions of the patients with sALS supports the concept of relatively reduced inhibitory GABAergic influence as a potential mechanism accounting for, or exacerbating, increased cortical excitability. We propose that the homozygous D90A patients have a distinct cortical vulnerability, one in which intrinsic cortical inhibitory neurones are relatively preserved, ${ }^{19}$ and which may have a bearing on the latter group's characteristic and slowly progressive disease phenotype.

\section{ACKNOWLEDGEMENTS}

M R Turner is supported by a Wellcome Trust Clinical Research Fellowship, who also provide funding to A D Osei-Lah and K R Mills. The King's MND Care \& Research Centre (M R Turner, A Al-Chalabi, C E Shaw, P N Leigh) receives support from the Motor Neurone Disease Association (UK). A Hammers receives support from the Medical Research Council (MRC). A Al-Chalabi is an MRC Clinician Scientist. P M Andersen is supported by the Swedish Brain Research Foundation, the Hållstens Brain Research Foundation, and the Swedish Medical Society and the Björklund Foundation for ALS Research. D J Brooks receives support from the MRC.
We are indebted to the patients for their participation in this project, particularly those who travelled to the UK from Sweden. Thanks also to the radiographers A Blyth, H McDevitt and S Ahier, and PET technician L Scnorr.

\section{Authors' affiliations}

M R Turner, A Al-Chalabi, P N Leigh, Department of Neurology, PO Box 41, Institute of Psychiatry, De Crespigny Park, London SE5 8AF, UK M R Turner, A Hammers, D J Brooks, MRC Clinical Sciences Centre and Division of Neuroscience, Faculty of Medicine, Imperial College, Hammersmith Hospital, Du Cane Road, London W12 ONN, UK A D Osei-Lah, K R Mills, Department of Clinical Neurophysiology, Guy's, King's \& St. Thomas's School of Medicine, Academic Neuroscience Centre, King's College Hospital, London SE5 9RS, UK

A Hammers, D J Brooks, Institute of Neurology, Queen Square, London WCIN 3BG, UK

C E Shaw, Department of Neurology, Guy's, King's \& St. Thomas's School of Medicine, Academic Neuroscience Centre, King's College Hospital, London, SE5 9RS, UK

P M Andersen, Department of Neurology, Umeå University Hospital, Umeå, Sweden

Competing interests: none declared

\section{REFERENCES}

1 Rowland LP, Shneider NA. Amyotrophic lateral sclerosis. N Engl J Med 2001;344:1688-1700.

2 Smith MC. Nerve fibre degeneration in the brain in amyotrophic lateral sclerosis. J Neurol Neurosurg Psychiatry 1960;23:269-82.

3 Maekawa S, Al Sarraj S, Kibble M, et al. Cortical selective vulnerability in Motor Neurone Disease: A morphometric study. Brain 2004; 127:1237-51

4 Turner MR, Cagnin A, Turkheimer FE, et al. Evidence of widespread cerebral microglial activation in amyotrophic lateral sclerosis: an [(11)C](R)-PK1 1195 positron emission tomography study. Neurobiol Dis 2004;15:601-9.

5 Mills KR. Magnetic stimulation of the human nervous system. Oxford: Oxford University Press, 1999

6 Rosler KM, Truffert A, Hess CW, et al. Quantification of upper motor neuron loss in amyotrophic lateral sclerosis. Clin Neurophysiol 2000;111:2208-18.

7 Mills KR. Inhibitory effects. In: Mills KR, ed. Magnetic stimulation of the human nervous system. Oxford: Oxford University Press, 1999:87-98.

8 Yokota T, Yoshino A, Inaba A, et al. Double cortical stimulation in amyotrophic lateral sclerosis. J Neurol Neurosurg Psychiatry 1996;61:596-600.

9 Ziemann U, Winter M, Reimers CD, et al. Impaired motor cortex inhibition in patients with amyotrophic lateral sclerosis. Evidence from paired transcranial magnetic stimulation. Neurology 1997;49:1292-8.

10 Hanajima R, Ugawa Y. Impaired motor cortex inhibition in patients with ALS: evidence from paired transcranial magnetic stimulation. Neurology 1998;51:1771-2.

11 Leigh PN, Meldrum BS. Excitotoxicity in ALS. Neurology 1996:47(suppl 4):S221-7.

12 Lloyd CM, Richardson MP, Brooks DJ, et al. Extramotor involvement in ALS: PET studies with the GABA(A) ligand [ ( 111$)$ C]flumazenil. Brain 2000; 123:2289-96.

13 Rosen DR, Siddique T, Patterson D, et al. Mutations in Cu/Zn superoxide dismutase gene are associated with familial amyotrophic lateral sclerosis. Nature 1993;362:59-62.

14 Andersen PM, Sims KB, Xin WW, et al. Sixteen novel mutations in the Cu/Zn superoxide dismutase gene in amyotrophic lateral sclerosis: a decade of discoveries, defects and disputes. Amyotroph Lateral Scler Other Motor Neuron Disord 2003;4:62-73.

15 Andersen PM. Genetic aspects of amyotrophic lateral sclerosis/motor neuron disease. In: Shaw PJ, Strong MJ, eds. Motor neuron disease. Butterworth Heinemann, 2003:207-36.

16 Andersen PM, Forsgren L, Binzer M, et al. Autosomal recessive adult-onset amyotrophic lateral sclerosis associated with homozygosity for Asp90Ala CuZn-superoxide dismutase mutation. A clinical and genealogical study of 36 patients. Brain 1996;119:1153-72.

17 Al-Chalabi A, Andersen PM, Chioza B, et al. Recessive amyotrophic lateral sclerosis families with the D9OA SOD1 mutation share a common founder: evidence for a linked protective factor. Hum Mol Genet 1998;7:2045-50.

18 Parton MJ, Broom W, Andersen PM, et al. D90A-SOD1 mediated amyotrophic lateral sclerosis: a single founder for all cases with evidence for a cis-acting disease modifier in the recessive haplotype. Hum Mutat 2002;20:473.

19 Weber M, Eisen A, Stewart HG, et al. Preserved slow conducting corticomotoneuronal projections in amyotrophic lateral sclerosis with autosomal recessive D90A CuZn-superoxide dismutase mutation. Brain 2000;123:1505-15.

20 Brooks BR, Miller RG, Swash M, et al. El Escorial revisited: revised criteria for the diagnosis of amyotrophic lateral sclerosis. Amyotroph Lateral Scler Other Motor Neuron Disord 2000;1:293-9. 
21 Cedarbaum JM, Stambler N, Malta E, et al. The ALSFRS-R: a revised ALS functional rating scale that incorporates assessments of respiratory function. BDNF ALS Study Group (Phase III). J Neurol Sci 1999;169:13-21.

22 Mills KR, Nithi KA. Corticomotor threshold is reduced in early sporadic amyotrophic lateral sclerosis. Muscle Nerve 1997;20:1137-41.

23 Rossini PM, Marciani MG, Caramia M, et al. Nervous propagation along 'central' motor pathways in intact man: characteristics of motor responses to 'bifocal' and 'unifocal' spine and scalp non-invasive stimulation. Electroencephalogr Clin Neurophysiol 1985;61:272-86.

24 Cunningham VJ, Jones T. Spectral analysis of dynamic PET studies. J Cereb Blood Flow Metab 1993;13:15-23.

25 Friston KJ, Frith CD, Liddle PF, et al. Comparing functional (PET) images: the assessment of significant change. J Cereb Blood Flow Metab 1991; 11:690-9

26 Doble A. The role of excitotoxicity in neurodegenerative disease: implications for therapy. Pharmacol Ther 1999:81:163-221.

27 Petri S, Krampfl K, Hashemi F, et al. Distribution of GABAA receptor mRNA in the motor cortex of ALS patients. J Neuropathol Exp Neurol 2003;62:1041-51

28 Kew JJ, Leigh PN, Playford ED, et al. Cortical function in amyotrophic lateral sclerosis. A positron emission tomography study. Brain 1993;116:655-80.

29 Sommer M, Tergau F, Wischer S, et al. Riluzole does not have an acute effect on motor thresholds and the intracortical excitability in amyotrophic lateral sclerosis. J Neurol 1999;246(suppl 3):III22-6.

30 Desiato MT, Palmieri MG, Giacomini P, et al. The effect of riluzole in amyotrophic lateral sclerosis: a study with cortical stimulation. J Neurol Sci 1999; 169:98-107.

31 Stefan K, Kunesch E, Benecke R, et al. Effects of riluzole on cortical excitability in patients with amyotrophic lateral sclerosis. Ann Neurol 2001;49:536-9.

32 Liepert J, Schwenkreis P, Tegenthoff $M$, et al. The glutamate antagonist riluzole suppresses intracortical facilitation. J Neural Transm 1997; 104:1207-14

33 Schwenkreis $\mathbf{P}$, Liepert J, Witscher K, et al. Riluzole suppresses motor cortex facilitation in correlation to its plasma level. A study using transcranial magnetic stimulation. Exp Brain Res 2000;135:293-9.

34 Aventis Pharmaceuticals Inc. Rilutek (riluzole) tablets prescribing information, 2003; 35. www.aventispharma-us.com/PIs/rilutek_txt.html.

35 Hanajima R, Ugawa Y, Terao Y, et al. Ipsilateral cortico-cortical inhibition of the motor cortex in various neurological disorders. J Neurol Sci 1996;140:109-16.
36 Turazzini M, Manganotti P, Del Colle R, et al Serum levels of carbamazepine and cortical excitability by magnetic brain stimulation. Neurol Sci 2004;25:83-90.

37 Davison C. Amyotrophic lateral sclerosis. Origin and extent of the upper motor neuron lesion. Arch Neurol Psychiatr 1941;46:1036-56.

38 Peretti-Viton P, Azulay JP, Trefouret S, et al. MRI of the intracranial corticospinal tracts in amyotrophic and primary lateral sclerosis. Neuroradiology 1999;41:744-9.

39 Mills KR. The natural history of central motor abnormalities in amyotrophic lateral sclerosis. Brain 2003;126:2558-66.

40 Eisen A, Shytbel W, Murphy K, et al. Cortical magnetic stimulation in amyotrophic lateral sclerosis. Muscle Nerve 1990;13:146-51.

41 Desiato MT, Caramia MD. Towards a neurophysiological marker of amyotrophic lateral sclerosis as revealed by changes in cortical excitability. Electroencephalogr Clin Neurophysiol 1997;105:1-7.

42 Mills KR, Nithi KA. Peripheral and central motor conduction in amyotrophic lateral sclerosis. J Neurol Sci 1998;159:82-7.

43 Osei-Lah AD, Turner MR, Andersen PM, et al. A novel central motor conduction abnormality in D90A-homozygous patients with amyotrophic lateral sclerosis. Muscle Nerve 2004;29:790-4.

44 Eisen A, Pant B, Stewart H. Cortical excitability in amyotrophic lateral sclerosis: a clue to pathogenesis. Can J Neurol Sci 1993;20:11-16.

45 De Carvalho M, Evangelista T, Sales-Luis ML. The corticomotor threshold is not dependent on disease duration in amyotrophic lateral sclerosis (ALS) Amyotroph Lateral Scler Other Motor Neuron Disord 2002;3:39-42.

46 Prout AJ, Eisen AA. The cortical silent period and amyotrophic lateral sclerosis. Muscle Nerve 1994;17:217-23.

47 Triggs WJ, Menkes D, Onorato J, et al. Transcranial magnetic stimulation identifies upper motor neuron involvement in motor neuron disease. Neurology 1999;53:605-1.

48 De Carvalho M, Turkman A, Swash M. Motor responses evoked by transcranial magnetic stimulation and peripheral nerve stimulation in the ulnar innervation in amyotrophic lateral sclerosis: the effect of upper and lower motor neuron lesion. J Neurol Sci 2003;210:83-90.

49 Ellis CM, Simmons A, Jones DK, et al. Diffusion tensor MRI assesses corticospinal tract damage in ALS. Neurology 1999;53:1051-8.

50 Cahn SD, Herzog AG, Pascual-Leone A. Paired-pulse transcranial magnetic stimulation: effects of hemispheric laterality, gender, and handedness in normal controls. J Clin Neurophysiol 2003;20:371-4. 